\title{
PRODUTOS DE ACEROLA: ESTUDO DA ESTABILIDADE DE VITAMINA ${ }^{1}$
}

\author{
Fábio YAMASHITA ${ }^{2, *}$; Marta de Toledo BENASSI²; Anamaria Caldo TONZAR ${ }^{3}$; \\ Suely MORIYA3; Joicelena Georgetti FERNANDES ${ }^{3}$
}

\begin{abstract}
RESUMO
A acerola é um fruto altamente perecivel e que contém altos teores de vitamina C, sendo este o seu principal atrativo em termos nutricionais. A atual legislação brasileira prevê uma variação de, no máximo, $20 \%$ do teor dos nutrientes especificados no rótulo. Devido a essa exigência seria importante que os fabricantes considerassem tanto o teor inicial de vitamina $\mathrm{C}$ quanto a perda ao longo da armazenagem dos produtos de acerola. Neste trabalho, foi feito o acompanhamento da estabilidade da vitamina $\mathrm{C}$ em polpa pasteurizada e acerola in natura congeladas, ambas armazenadas a $-12^{\circ} \mathrm{C} \mathrm{e}-18^{\circ} \mathrm{C}$, e em suco de acerola pasteurizado engarrafado, mantido a temperatura ambiente, ao longo de 4 meses de armazenagem. As polpas congeladas não apresentaram degradação significativa durante este periodo, já as in natura apresentaram cinética de degradação de $1^{\mathrm{a}}$ ordem e o suco de ordem zero. Após 4 meses de armazenagem as acerolas armazenadas a $-12^{\circ} \mathrm{C} \mathrm{e}-18^{\circ} \mathrm{C}$ apresentaram teores de $869 \pm 12$ e $1.223 \pm 148 \mathrm{mg}$ vit. $\mathrm{C} / 100 \mathrm{~g}$, representando uma perda de $43 \%$ e $19 \%$, respectivamente, em relação ao teor inicial. Polpas a $-12^{\circ} \mathrm{C}$ e $-18^{\circ} \mathrm{C}$ apresentaram teores de $1.314 \pm 6$ e $1.322 \pm 2 \mathrm{mg}$ vit. C/ $100 \mathrm{~g}$, respectivamente, representando uma perda de, aproximadamente, $3 \%$ e o suco apresentou uma perda de $32 \%$, correspondendo a um teor final de $673 \pm 17 \mathrm{mg}$ vit.C/100g.

Palavras-chave: armazenagem; congelamento; suco; polpa; frutas; rotulagem; cinética.
\end{abstract}

\section{SUMMARY}

WEST INDIAN CHERRY PRODUCTS: STUDY OF VITAMIN C STABILITY. West indian cherry is a very perishable fruit and its vitamin C content is its principal nutritional appeal. Brazilian legislation admits a $20 \%$ variation in the nutrient content from the one specified on the label. Due to this requirement the initial vitamin C content and losses during storage should be known in products. Vitamin $\mathrm{C}$ stability in pasteurized pulp and west indian cherry in natura, both frozen and stored at $-12^{\circ} \mathrm{C} \mathrm{e}-18^{\circ} \mathrm{C}$ and of the pasteurized juice stored at room temperature, were determined during four months. Frozen pulps had no significant vitamin $\mathrm{C}$ degradation during this period and fruits in natura showed first order kinetics degradation and zero order for the juice. After four months, west indian cherry fruits stored at $-12^{\circ} \mathrm{C}$ and $-18^{\circ} \mathrm{C}$ showed $869 \pm 12$ and $1.223 \pm 148 \mathrm{mg}$ vit.C/100g, representing losses of $43 \%$ and $19 \%$, respectively. Pulps at $-12^{\circ} \mathrm{C}$ and $-18^{\circ} \mathrm{C}$ showed a $1.314 \pm 6$ and $1.322 \pm 2 \mathrm{mg}$ vit. $\mathrm{C} / 100 \mathrm{~g}$, respectively, representing approx. $3 \%$ losses and the juice showed $32 \%$ loss, corresponding to a final content of $673 \pm 17 \mathrm{mg}$ vit.C/100g.

Keywords: storage; freezing; juice; pulp; fruit; labeling; kinetics.

\section{1 - INTRODUÇÃO}

A acerola ou cereja das Antilhas (Malpighia glabra L., Malpighia punicifolia L. ou Malphigia emarginata DC.) é originária da América Tropical e seu principal atrativo é o alto teor de vitamina $\mathrm{C}$, sendo também rica em outros nutrientes como carotenóides, tiamina, riboflavina e niacina [2]. No mercado, encontram-se vários produtos alimentícios de acerola, sendo as formas mais comuns de comercialização a acerola in natura e polpa congeladas e suco engarrafado. Os prazos de validade desses produtos, de acordo com os fabricantes, variam de 4 a 12 meses.

A resolução RDC no 40 de março de 2001 [5] prevê que a rotulagem nutricional passa a ser obrigatória para todos os alimentos e bebidas embaladas e que as vitaminas somente serão declaradas quando se encontrarem presentes em pelo menos $5 \%$ da ingestão diária recomendada (IDR), por porção, sendo permitida uma variação de no máximo $20 \%$ do valor especificado no rótulo. No caso dos produtos de acerola, os fabricantes deveriam saber o teor inicial e quanto se perde de vitamina $\mathrm{C}$

\footnotetext{
1. Recebido para publicação em 20/09/2001. Aceito para publicação em 15/05/2002.

${ }^{2}$ UNIVERSIDADE ESTADUAL DE LONDRINA - Depto. Tecnologia de Alimentos e Medicamentos, Centro de Ciências Agrárias, C.P. 6001, CEP 86051-970, Londrina - PR. fabioy@uel.br-Tel (43) 371-4565 ramal 207 - Fax (43) 371-4080

${ }^{3}$ UNESP - DETA. C.P. 136, CEP 15054-O0O, São José do Rio Preto-SP * A quem a correspondência deve ser enviada.
}

ao longo da armazenagem para estimar o teor no final da vida-de-prateleira do produto e adequá-lo a rotulagem.

O objetivo do trabalho foi determinar a estabilidade da vitamina $\mathrm{C}$ em acerola in natura e polpa pasteurizada, ambas congeladas a $-12^{\circ} \mathrm{C}$ e $-18^{\circ} \mathrm{C}$, e de suco de acerola pasteurizado engarrafado, mantido à temperatura ambiente, ao longo de 4 meses de armazenagem. As condições de temperatura escolhidas simulam as condições comerciais $\left(-18^{\circ} \mathrm{C}\right)$ e domésticas $\left(-12^{\circ} \mathrm{C}\right)$ de armazenagem do produto.

\section{2 - MATERIAL E MÉTODOS}

\section{1 - Material}

Foram utilizadas 100 embalagens plásticas contendo $50 \mathrm{~g}$ de polpa de acerola pasteurizada congelada, 12 pacotes de $500 \mathrm{~g}$ contendo acerola in natura congelada e 24 garrafas contendo suco de acerola reconstituído e pasteurizado e contendo como conservador ácido sórbico e seus sais, de acordo com o rótulo do produto. Todos os produtos foram adquiridos em supermercado, sendo que os congelados foram transportados em recipiente isotérmico e armazenados logo após a chegada ao laboratório. Todos os produtos foram provenientes dos mesmos lotes e com data de fabricação, no caso do suco, de 13 dias anterior ao inicio dos experimentos, da acerola congelada de 9 dias e da polpa de 12 dias. 


\section{2 - Métodos}

Foram determinados os teores iniciais de ácido ascórbico do suco, da polpa e da acerola in natura antes do inicio da armazenagem e a cada 30 dias, num total de 4 meses. As amostras foram armazenadas no congelador de um refrigerador doméstico de um corpo, marca Consul, com temperatura de $-12^{\circ} \mathrm{C} \pm 2^{\circ} \mathrm{C}$ e num congelador horizontal marca Prosdócimo, com temperatura de $18^{\circ} \mathrm{C} \pm 2^{\circ} \mathrm{C}$.

Foram feitas análises do teor de sólidos solúveis totais (SST) como ${ }^{\circ}$ Brix e da acidez total titulável (ATT) do produto, ao longo da armazenagem, de acordo com as normas analiticas do INSTITUTO ADOLFO LUTZ [6]. A leitura de SST foi corrigida de acordo com a ATT e a temperatura [6].

Para a determinação do teor de vitamina $\mathrm{C}$ foi utilizado o método padrão da AOAC [1], modificado por BENASSI e ANTUNES [3]. Amostras de $2 \mathrm{~g}$ de acerola foram homogeneizadas com $50 \mathrm{~g}$ de solução extratora (ácido oxálico $2 \%)$ em liquidificador por dois minutos. Uma alíquota de $20 \mathrm{~g}$ foi tomada e diluída com a mesma solução empregada para extração para $50 \mathrm{~mL}$ em balão volumétrico. Uma alíquota de $10 \mathrm{~mL}$ dessa solução foi titulada com solução padronizada de 2,6-diclorofenolindofenol 0,01\%, sendo o ponto de viragem detectado visualmente. Todas as análises foram feitas em duplicata.

Com os valores do teor de vitamina $\mathrm{C}$ pelo tempo de armazenagem, no caso de cinética de degradação de ordem zero, ou do logaritmo neperiano do teor, no caso de cinética de $1^{a}$ ordem, foram calculadas as taxas de degradação de vitamina $C$, tempo de meia-vida, $Q_{10}$ e energia de ativação, conforme LABUZA [8].

\section{3 - RESULTADOS}

\section{1 - Teores inicial e final de vitamina $\mathrm{C}$}

Os teores de vitamina $\mathrm{C}$ das acerolas congeladas in natura, da polpa pasteurizada congelada e do suco pasteurizado em garrafa, determinado no início do experimento, foram de $1.511 \pm 56,1.360 \pm 26$ e $988 \pm 50 \mathrm{mg}$ vit.C/ $100 \mathrm{~g}$ amostra, respectivamente, caracterizando a acerola como uma ótima fonte desta vitamina, mesmo após sofrer processamento térmico. No Brasil, a ingestão diária recomendada (IDR) de vitamina $\mathrm{C}$ para adultos é de $60 \mathrm{mg}$ [4]. ITOO et al. [7] encontraram teores mais altos para acerola in natura fresca (até $2.800 \mathrm{mg} / 100 \mathrm{~g}$ ) e suco recém-embalado (em torno de $2.000 \mathrm{mg} / 100 \mathrm{~g}$ ), sendo que estas diferenças estão ligadas principalmente à variedade e ao estádio de maturação do fruto, tipo de processamento e data da análise, pois as determinações não foram feitas imediatamente após a colheita ou processamento. Quanto à polpa congelada, os valores estão próximos aos obtidos por OLIVEIRA et al. [9], que levantou o teor de vitamina $\mathrm{C}$ de 28 marcas de polpas de acerola congeladas produzidas nos estados de Pernambuco e Paraíba e obteve um valor médio de $1.025 \pm 270 \mathrm{mg}$ vit.C/100g.

Após 4 meses de armazenagem, as acerolas armazenadas a $-12^{\circ} \mathrm{C} \mathrm{e}-18^{\circ} \mathrm{C}$ apresentaram teores de $869 \pm 12 \mathrm{e}$
$1.223 \pm 148 \mathrm{mg}$ vit.C/ $100 \mathrm{~g}$, respectivamente, representando uma perda de $43 \%$ e $19 \%$, respectivamente, em relação ao teor inicial.

As amostras de polpa a $-12^{\circ} \mathrm{C} \mathrm{e}-18^{\circ} \mathrm{C}$ apresentaram teores de $1.314 \pm 6$ e $1.322 \pm 2 \mathrm{mg}$ vit.C/100g, respectivamente, representando uma pequena perda $(\sim 3 \%)$ após 4 meses de armazenagem.

O suco apresentou uma perda de $32 \%$ de vitamina após 4 meses de armazenagem, correspondendo a um teor final de $673 \pm 17 \mathrm{mg}$ vit.C $/ 100 \mathrm{~g}$. A literatura cita perdas em torno de 20 a $30 \%$ [7] para sucos de acerola armazenados durante 180 dias.

\section{2 - Influência do tipo de produto sobre a cinética de degradação de vitamina $C$}

Os teores de vitamina $\mathrm{C}$ nas acerolas in natura e polpas pasteurizadas, congeladas e armazenadas a $-12^{\circ} \mathrm{C} \mathrm{e}$ $-18^{\circ} \mathrm{C}$, e no suco de acerola pasteurizado, mantido a temperatura ambiente, estão na Figura 1.
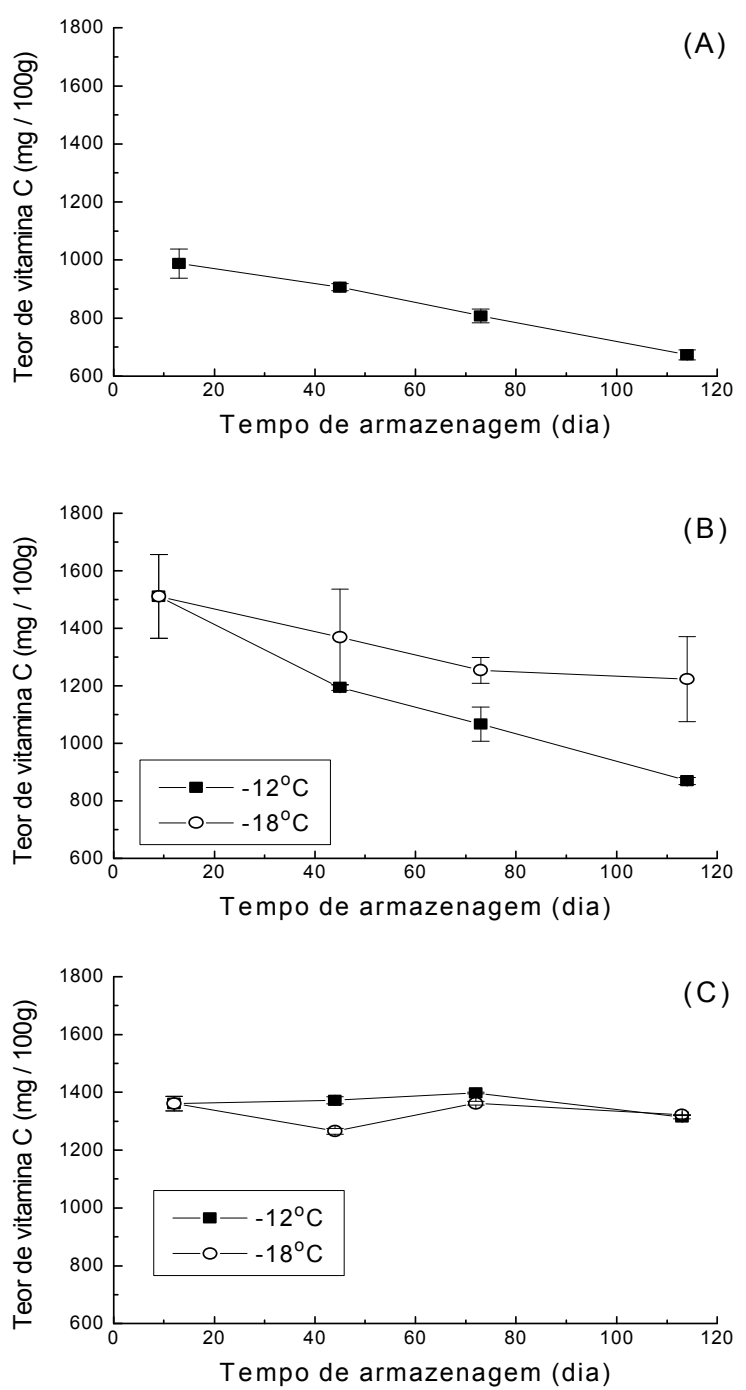

FIGURA 1. Variação do Teor de Vitamina $\mathrm{C}$ em Produtos de Acerola: suco (A) a temperatura ambiente, acerola in natura (B) e polpa (C) congeladas. 
O suco apresentou um decréscimo linear de vitamina $\mathrm{C}$ em função do tempo de armazenagem, característico de uma cinética de degradação de ordem zero (Tabela 1), com um tempo de meia-vida de 164 dias. Este comportamento não tem sido reportado como característico de vitamina $\mathrm{C}$, que em geral apresenta cinética de degradação de primeira ordem [10].

Já a acerola in natura, apresentou uma cinética de degradação de primeira ordem, com tempos de meia-vida altos, sendo que a taxa de degradação das armazenadas $\mathrm{a}-12^{\circ} \mathrm{C}$ foi 2,4 vezes maior que a $-18^{\circ} \mathrm{C}$ (Tabela 1$)$. Portanto, a temperatura de armazenagem é importante para este tipo de produto, uma vez que, por não ter sido submetido a pasteurização, a atividade enzimática não foi paralisada. O valor de $Q_{10}$ calculado foi de 4,2, com uma energia de ativação de $18.854 \mathrm{kcal} / \mathrm{kg} . \mathrm{mol}$, semelhante ao obtido para degradação de vitamina $\mathrm{C}$ em manga (17.237kcal/kg.mol) [11].

A polpa congelada manteve o teor de vitamina C praticamente constante $(1.344 \pm 42 \mathrm{mg}$ vit. $\mathrm{C} / 100 \mathrm{~g})$ ao longo da armazenagem, tanto a $-12^{\circ} \mathrm{C}$ como a $-18^{\circ} \mathrm{C}$ (Tabela 1$)$. Neste caso, como a polpa foi pasteurizada e mantida a baixas temperaturas, não houve diferença entre as temperaturas de armazenagem em relação à degradação da vitamina.

TABELA 1. Taxa de Degradação de Vitamina $\mathrm{C}\left(\mathrm{N}_{\mathrm{vc}}\right)$, Tempo de Meia-Vida $\left(\mathrm{t}_{1 / 2}\right)$, Coeficiente de Determinação $\left(\mathrm{r}^{2}\right)$, Número de Amostras (N) e Nivel de Significância das Correlações (P) para Degradação de Vitamina C em Diversos Produtos de Acerola

\begin{tabular}{|c|c|c|c|c|c|c|c|}
\hline 'roduto & $\begin{array}{l}\text { Ordem da } \\
\text { reação }\end{array}$ & $\begin{array}{l}\text { Temp. } \\
\left({ }^{\circ} \mathrm{C}\right)\end{array}$ & $\begin{array}{l}\mathrm{N}_{\mathrm{vc}} \quad(\mathrm{mg} \\
\text { vit } \mathrm{C} / 100 \mathrm{~g} \text {.dia) }\end{array}$ & $\mathrm{t}_{1 / 2}$ (dia) & $\mathrm{R}^{2}$ & $\mathrm{~N}$ & $\mathrm{p}$ \\
\hline natura" & $1^{\underline{a}}$ & -12 & $5,41 \times 10^{-3}$ & 1.229 & 0,91 & 14 & $<0,01$ \\
\hline natura" & $1^{\underline{a}}$ & -18 & $2,30 \times 10^{-3}$ & 2.881 & 0,54 & 14 & $<0,01$ \\
\hline Suco & Zero & Ambiente & 3,15 & 164 & 0,97 & 8 & $<0,01$ \\
\hline
\end{tabular}

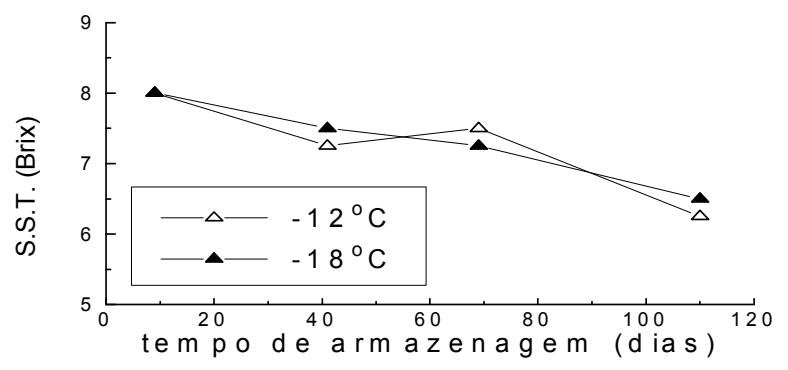

FIGURA 2. Variação do teor de sólidos solúveis totais em acerola in natura congelada

Nos produtos que sofreram tratamento térmico (suco engarrafado e polpa congelada a $-12^{\circ} \mathrm{C} \mathrm{e}-18^{\circ} \mathrm{C}$ ), os teores de sólidos solúveis totais em ${ }^{\circ} \mathrm{Brix}$ (SST) mantiveram-se praticamente constantes ao longo dos 4 meses de armazenagem $\left(5,3 \pm 0,3,5,9 \pm 0,2\right.$ e $5,9 \pm 0,2^{\circ} \mathrm{Brix}$, respectivamente). Já o teor de SST da acerola in natura apre- sentou uma queda (Figura 2), indicando que o produto ainda apresentava atividade enzimática, mesmo em temperaturas de congelamento.

\section{4 - CONCLUSÕES}

A estabilidade de vitamina $\mathrm{C}$ em produtos de acerola foi dependente tanto do tipo de processamento quanto da temperatura de armazenagem, apresentando inclusive cinéticas de degradação diferenciadas (zero e primeira ordem). Os produtos que combinaram a pasteurização com congelamento apresentaram maior retenção de vitamina $\mathrm{C}$ ao final do período de armazenagem, independente da temperatura. Para acerola in natura, a temperatura de armazenagem seria um fator importante a ser considerado, uma vez que as taxas de degradação foram 2,4 vezes maiores a $-12^{\circ} \mathrm{C}$ que a $-18^{\circ} \mathrm{C}$. Dos produtos testados, o suco foi o que apresentou o menor tempo de meia-vida, apenas 164 dias.

\section{5 - REFERÊNCIAS}

[1] AOAC. Official methods of analysis. Association of Official Analytical Chemists, Washington D. C., 1984. p. 844-845.

[2] ASSIS, S.A.; LIMA, D.C.; OLIVEIRA, O.M.M.F. Activity of pectinmethylesterase, pectin content and vitamin $C$ in acerola fruit at various stages of fruit development. Food Chemistry, v.74, p.133-137, 2001.

[3] BENASSI, M. T.; ANTUNES, A. J. A comparison of metaphosphoric and oxalic acids as extractant solutions for the determination of vitamin $\mathrm{C}$ in selected vegetables. Arquivos de Biologia e Tecnologia, v.31, n.4, p.507513, 1988.

[4] BRASIL. Portaria SVS/MS n 33, de 13 de janeiro de 1998. Tabelas de Ingestão Diária Recomendada (IDR). Diário Oficial da União de 16 de janeiro de 1998.

[5] BRASIL. Resolução RDC no 40, Agência Nacional de Vigilância Sanitária, de 21 de março de 2001. Rotulagem Nutricional Obrigatória. Diário Oficial da União de 22 de março de 2001.

[6] INSTITUTO ADOLFO LUTZ. Normas analiticas do Instituto Adolfo Lutz. 2 ed. Instituto Adolfo Lutz, São Paulo, 1976. v.1.

[7] ITOO, S., AIBA, M. \& ISHIHATA, K. Ascorbic acid content in acerola fruit from different production regions and degrees of maturity, and stability during processing. Journal of Japanese Society of Food Science and Technology, v.37, n.9, p.726-729, 1990.

[8] LABUZA, T.P. Shelf-life dating of foods. Food \& Nutrition Press Inc., Westport, 1982.

[9] OLIVEIRA, M.E.B.; BASTOS, M.S.R.; FEITOSA, T.; BRANCO, M.A.A.C.; SILVA, M.G.G. Avaliação de parâmetros de qualidade fisico-químicos de polpas congeladas de acerola, cajá e caju. Ciênc. e Tecnol. de Aliment., v.19, n.3, p.326-332, 1999.

[10] VILLOTA, R.; HAWKES, J.G. Reaction kinetics in food systems. In: HELDMAN, D.R.; LUND, D.B. (Ed.) Handbook of food engineering. Marcel Dekker, Inc., New York, 1992. p.39-144.

[11] YAMASHITA, F.; BENASSI, M.T.; KIECKBUSCH, T.G. Effect of modified atmosphere packaging on kinetics of vitamin $\mathrm{C}$ degradation in mangos. Brazilian Journal of Food Technology, v.2, n.1, p.127-130, 1999. 